\title{
Fluoreszente Biosensorik in Mikrofluidikchips mit integrierten nanoporösen Glasmembranen
}

\author{
E. Pöhler, S. Nagl \\ Universität Leipzig, Linnéstraße 304103 Leipzig, Deutschland \\ elisabeth.poehler@uni-leipzig.de
}

\begin{abstract}
Kurzfassung
\end{abstract}
Im Rahmen dieser Arbeit wurden fluoreszente pH-Sensormatrizen in mikrofluidische Reaktoren integriert und deren $\mathrm{pH}$-Sensitivität untersucht. Zur on-chip pH-Detektion wurde die Methode des Dual Lifetime Referencing (DLR) im mikroskopischen Maßstab basierend auf ratiometrischer Aufnahme von Fluoreszenzlebenszeiten mithilfe geeigneter Indikator- und Referenznanopartikel im Mikromaßstab angewandt. Dafür wurde die Sensormatrix mittels Rotationsbeschichtung in einen GlasPolymer Hybridchip eingebracht. Darüber hinaus wurde das Sensorsystem kovalent an eine nanoporöse Glasmembran gebunden, um deren vorteilhafte Oberflächenbeschaffenheit zu nutzen. Die Mikrochips sollen zur Entwicklung eines enzymatischen Mikrobiosensors verwendet werden, welcher zur schnellen und mobilen Überwachung von Pestiziden über die lokale pH-Änderung geeignet ist.

Schlüsselwörter: fluoreszenter pH Sensor, Mikroreaktor, Lumineszenzlebenszeitmessung, indikatorversetzte Nanopartikel, nanoporöse Glasmembran.

\section{Einleitung}

Die Verwendung von Pestiziden auf und in Lebensmitteln wird durch zahlreiche EUVorschriften geregelt. Diese mitunter hochtoxischen Substanzen sind dennoch sehr stark verbreitet. Die Aufnahme in den menschlichen Körper kann über verschiedene Wege, wie beispielsweise durch Inhalation, Hautkontakt oder über die Nahrungsaufnahme, erfolgen. Aufgrund dessen sind die Kontrolle und Einhaltung von bestimmten Grenzwerten für Pestizide und deren Rückstände unerlässlich. Bisher erfolgten diese Kontrollen mithilfe zeitaufwendiger und kostenintensiver Messverfahren. Da viele Pestizide, welche für den Menschen ein besonderes Gefahrenpotential darstellen, auf der Inhibition des Enzyms Acetylcholinesterase (AChE) basieren, sind enzymatische Sensoren von großer Bedeutung auf dem Weg zur schnellen und lokalen Überwachung von Pestiziden. Enzymatische Biosensoren nutzen die evolutionär entwickelte Selektivität natürlicher oder biotechnologisch hergestellter Makromoleküle für die Echtzeitanalytik vieler Substanzen und sind deshalb unter anderem für die flexible Umwelt- und Nahrungsmittelüberwachung von großem Interesse. Die auf das Enzym AChE basierenden Biosensoren nutzen die Änderung des $\mathrm{pH}-$ Wertes als Folge der Inhibition. Diese $\mathrm{pH}$-Änderung kann online und in Echtzeit mithilfe optischer, fluoreszenter Sensoren detektiert werden. Die Untersuchung des lokalen pH-Wertes mittels solcher Sensoren ermöglicht eine hohe räumliche Auflösung und Präzision. Die Miniaturisierung fluoreszenter Sensoren bietet dabei verschiedene Vorteile. Allen voran steht der geringe Verbrauch an Reagenzien und daraus resultierend eine effiziente Ökonomie. Weiterhin können, durch Systemintegration und Miniaturisierung in Mikrochips, die Aufarbeitungs- und Analysezeiten von Umweltproben deutlich verkürzt werden. Ein weiterer Vorteil liegt auch in der potenziellen Portabilität eines solchen Analysesystems. Hierzu sind jedoch Herausforderungen in der Fabrikations- und Detektionstechnologie zu bewältigen, um eine effiziente Analytik im Mikromaßstab durchführen zu können.

\section{Sensorpräparation}

Zur ratiometrischen $\mathrm{pH}$-Detektion wurden zwei verschiedene Lumineszenzfarbstoffe verwendet. Da das Messprinzip auf der Referenzierung der Farbstoffe zueinander beruht, müssen diese bestimmte Anforderungen erfüllen. Dafür wird ein kurzlebiger, $\mathrm{pH}$-sensitiver Fluorophor benötigt, dessen Emissionsintensität bzw. Lumineszenzlebenszeit zu einem langlebigen, nicht $\mathrm{pH}$-sensitiven Fluorophor referenziert wird [1]. Eine weitere Voraussetzung ist die spektrale Überlappung, um eine gleichzeitige Anregung und Detektion der beiden Fluorophore zu ermöglichen. Als $\mathrm{pH}$-sensitives Fluorophor wurde das Fluoresceinderivat 5-(6)Carboxyfluorescein verwendet, als 
Referenzfarbstoff

Ruthenium-trisphenanthrolin-dichlorid (Abb.1 a) und b)). Die Fluorophore wurden in nanopartikuläre Form gebracht. Das pH-sensitive Fluorophor wurde modifiziert und mithilfe von Tetraethylorthosilikat (TEOS) von einer Silikathülle umschlossen [2]. Der Referenzfarbstoff wurde in Polyacrylnitril (PAN) eingebracht [3]. Zur Herstellung der Beschichtungslösung wurden beide Partikeltypen jeweils in $1 \%$ (w/w) Konzentration in das Hydrogel Polyhydroxyethylmethacrylat (pHEMA) gebracht und in Ethanol/Wasser 9:1 $(w / w)$ in den mikrofluidischen Kanal eingebettet. Die Charakterisierung der Sensorschicht erfolgte mittels Britton-Robinson-Puffern (40 $\mathrm{mM} \mathrm{H} \mathrm{H}_{3} \mathrm{BO}_{3}, 40 \mathrm{mM} \mathrm{H} \mathrm{PO}_{4}$ und $40 \mathrm{mM}$ $\left.\mathrm{CH}_{3} \mathrm{COOH}, \mathrm{pH} 2-11\right)$. a

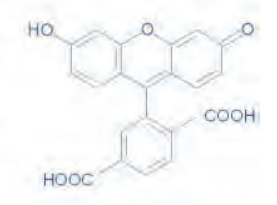

Carboxyfluorescein

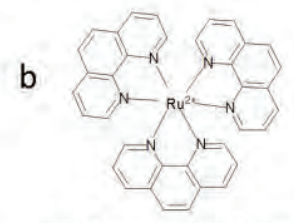

Tris(1,10-phenanthrolin) Ruthenium (II)

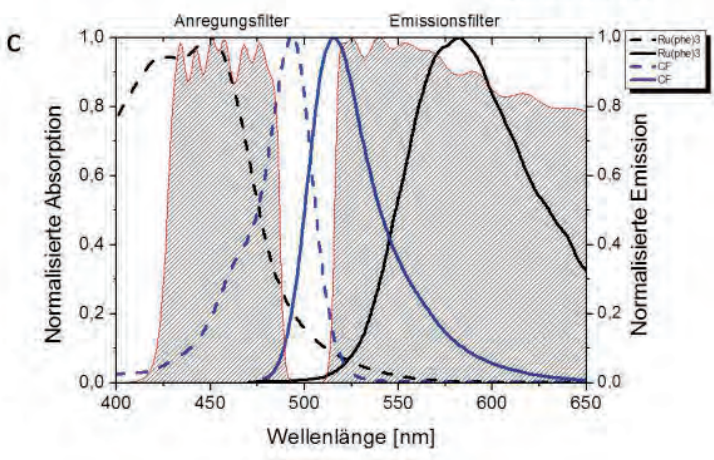

Abb. 1. Chemische Strukturen der beiden verwendeten Farbstoffe zur Nanopartikelherstellung a) $\mathrm{pH}$-sensitiver Fluorophor Carboxyfluorescein mit kurzer Fluoreszenzlebenszeit und b) Referenz (1,10phenanthrolin)Ruthenium (II) mit langlebiger Lumineszenzemission und c) Spektrum der beiden Farbstoffe mit den verwendeten Filtern.

\section{Experimenteller Aufbau}

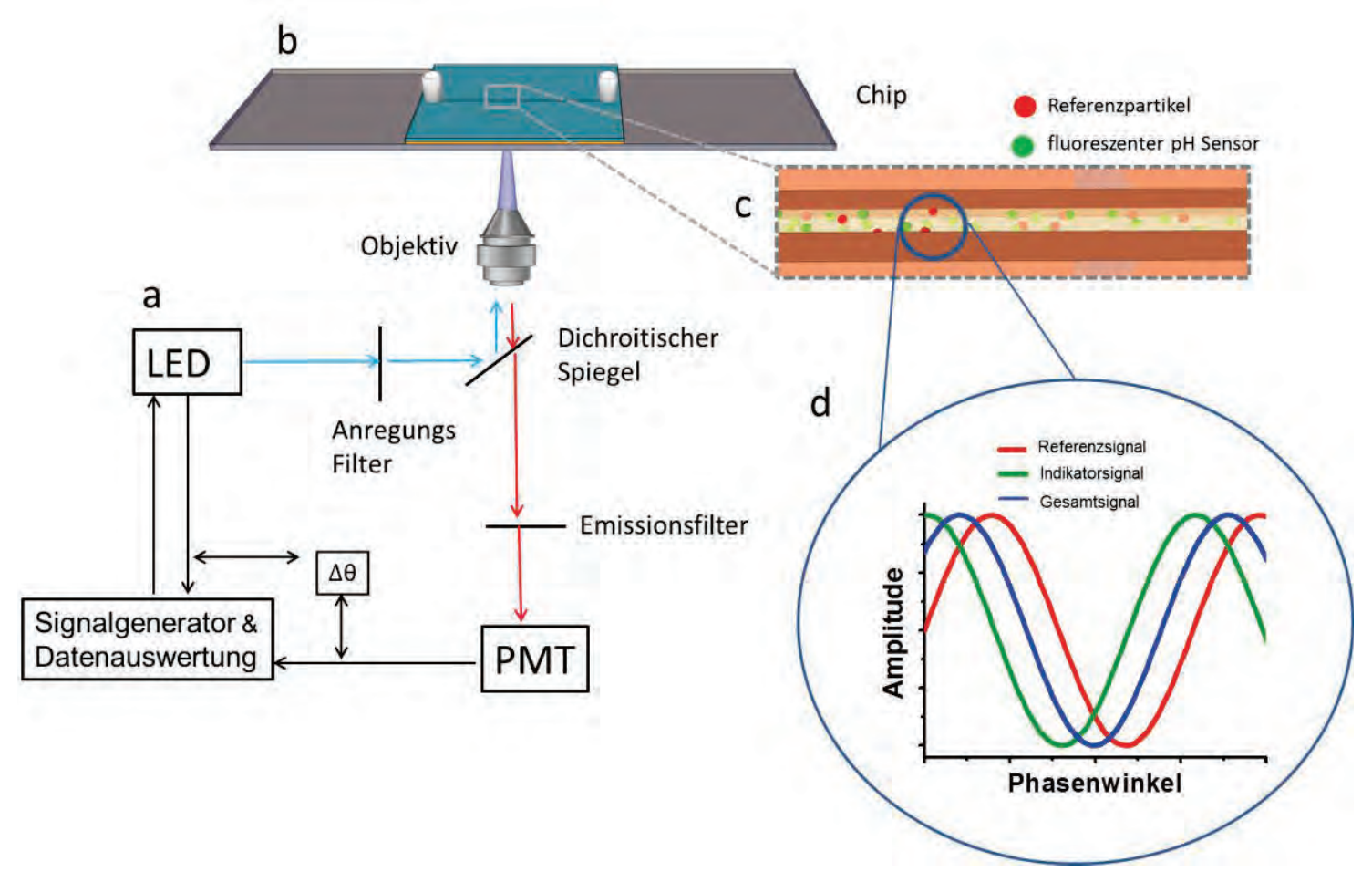

Abb. 2.: a) Schematische Zeichnung des Messaufbaus, b)Mikrofluidischer Chip zur pH Sensorik, c) Veranschaulichung des mikrofluidischen Kanals mit integrierten lumineszenten Nanopartikeln, d) Diagramm der Messmethodik

Für die sensitive und selektive Detektion der Mikrosensoren wurde ein Messaufbau entwickelt (Abb. 2). Dieser ermöglicht mittels LEDs als Lichtquellen, Photomultiplier (PMT) sowie eines Signalverstärkers mikroskopische
Lumineszenzlebenszeitmessungen in der Frequenzdomäne. 


\section{Ergebnisse}

Die lumineszenten Partikel wurden zunächst mit einer Konzentration von $1 \mathrm{mg} / \mathrm{mL}$ in eine Polymermatrix aus pHEMA eingebettet und mit Hilfe eines Fluorometers auf ihre pH-Sensitivität untersucht. Dazu wurde das dotierte Polymer auf eine PET-Trägerfolie aufgetragen welche in einer Küvette mit Britton-Robinson-Puffern unterschiedlicher pH-Werte versetzt wurde, (Abb. 3). Es konnte eine signifikante $\mathrm{pH}$ Abhängigkeit der Fluoreszenzintensität des Carboxyfluoresceins festgestellt werden, wohingegen die Fluoreszenzintensität des Ruthenium-Komplexes bei unterschiedlichen $\mathrm{pH}-$ Werten unveränderlich blieb.

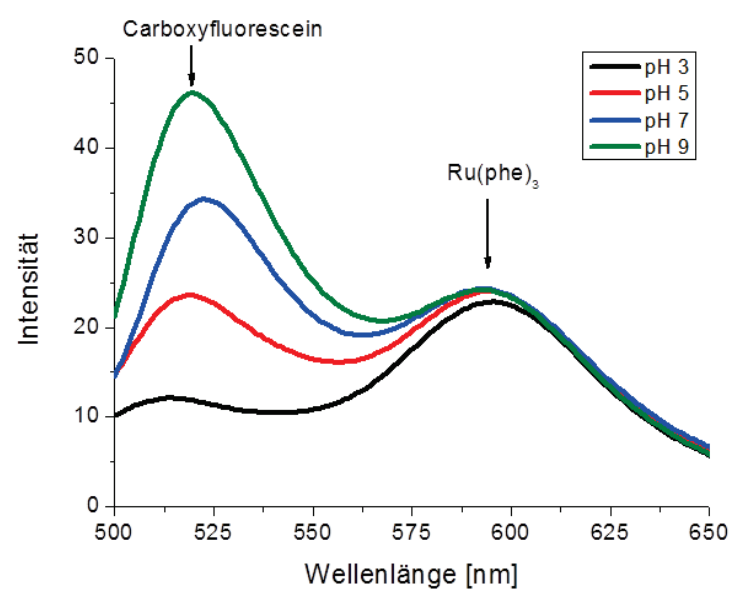

Abb 3. Fluoreszenzspektren der synthetisierten, lumineszenten Nanopartikel eingebettet in Polyhydroxyethylmethacrylat (pHEMA)

Die Nanopartikel in pHEMA wurden in ein photolithographisch hergestelltes mikrofluidisches System (Kanaldimension: 200 $\mu \mathrm{m} \times 100 \mu \mathrm{m})$, bestehend aus Glas und dem Photopolymer NOA81 gebracht (Abb. 2 b)). Dazu wurde der Mikrokanal mit einer Beschichtungsuspension, bestehend aus den Farbstoffpartikeln in 5\% pHEMA in einem 9:1 Ethanol/Wasser Gemisch, durch Kapillarkräfte befüllt. Nach Verdampfen des Lösungsmittels blieb das dotierte Polymer im Kanal zurück. Zur pH-Wert Messung wurde der mikrofluidische Kanal mit Britton-Robinson-Puffer unterschiedlicher pH-Werte durchspült und jeweils der Phasenwinkel bei einer Modulationsfrequenz von $45 \mathrm{kHz}$ aufgenommen. Wie in Abb. 4 ersichtlich, erfolgte eine starke Änderung des Phasenwinkels des emittierten Lichts der mikrochipintegrierten Sensorschicht von $27^{\circ}$ bei $\mathrm{pH} 3$ auf $18^{\circ}$ bei $\mathrm{pH} 10$. Der durch den $\mathrm{pK}_{\mathrm{S}^{-}}$ Wert der Sensormatrix definierte Arbeitsbereich des Mikro-pH-Sensors liegt im Bereich von ca. $\mathrm{pH} 5$ bis 9 mit einem $\mathrm{pK}_{\mathrm{s}}$ im Bereich des physiolologischen $\mathrm{pH}-$ Wertes bei 7,1.

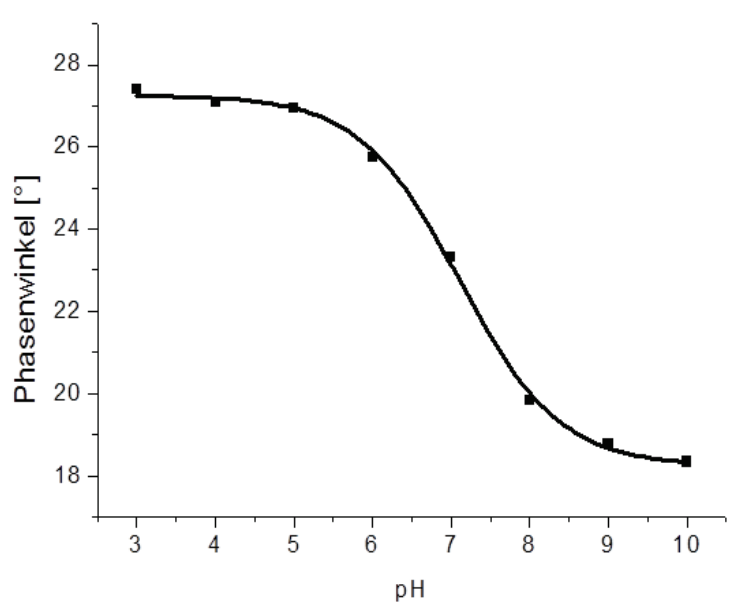

Abb 4. $\quad$ H-Abhängigkeitsuntersuchung des Phasenwinkels der Farbstoffpartikel in 5\% pHEMA , im Mikrochip. $\lambda_{\text {Exc: }}$ 420-480 nm, $\lambda_{E m}:>515 \mathrm{~nm}$

Als alternativer Ansatz wurden die für die DLRDetektion verwendeten Nanopartikel direkt auf einer in den Mikrochip integrierten nanoporösen Glasmembran (durchschnittliche Porengröße: $60 \mathrm{~nm}$ ) immobilisiert (Abb. 5). Dafür wurde sowohl die Membran als auch die lumineszenten Partikel mit Hilfe des Silanisierungsmittels Aminopropyltriethoxysilan oberflächenmodifiziert, um eine kovalente Anbindung zu ermöglichen. Die Anbindung erfolgte im Vakuum (Exsikkator: 600 mbar) für eine Stunde in PBS Puffer (phosphate buffered saline, $\mathrm{pH}$ $7,4)$.

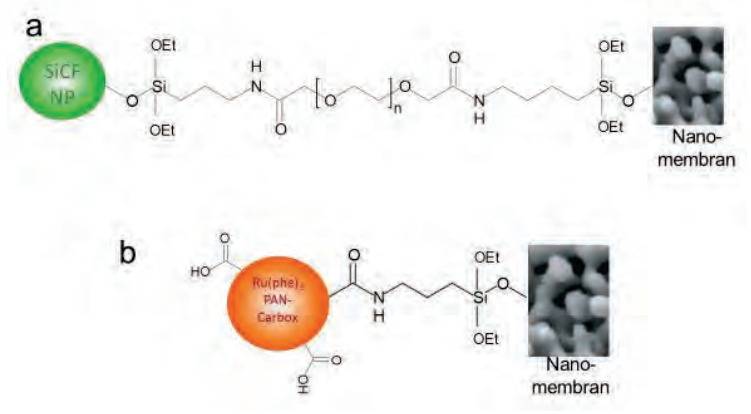

Abb 5. Modifikation der nanoporösen Glasmembran mit a) den $\mathrm{pH}$-sensitiven Partikeln (SICF NP)und b) den Referenzpartikeln (Ru(phe)3 PAN/Carbox)

Nach der kovalenten Anbindung beider Partikel auf einer Membran (Abb. 6a) wurde die Abhängigkeit des Phasenwinkels vom pH-Wert mit Britton-Robinson-Puffer untersucht (Abb. 6b). Die Flussrate betrug $200 \mu \mathrm{L} / \mathrm{min}$, die Anregungswellenlänge lag im Bereich von 420 bis $480 \mathrm{~nm}$ und die Emission wurde ab $515 \mathrm{~nm}$ aufgenommen .Der $\mathrm{pK}_{\mathrm{s}}-$ Wert des Systems wurde zu 6,22 festgestellt. 
a
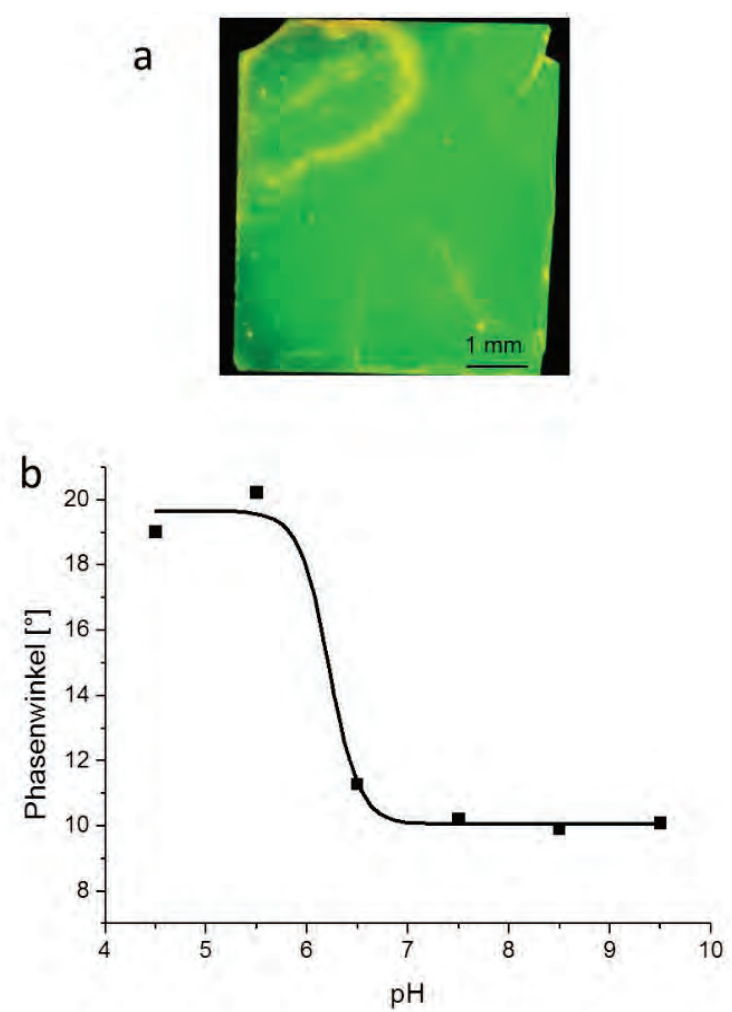

Abb 6. a) Fluoreszenzmikroskopische Aufnahme einer nanoporösen Glasmembran modifiziert mit $\mathrm{pH}$ sensorischen Nanopartikeln, b) $\mathrm{pH}$ Abhängigkeitsuntersuchung des Phasenwinkels hervorgerufen an einer modifizierten nanoporösen Glasmembran im Mikroreaktorchip (Modulationsfrequenz $45 \mathrm{kHz}, \lambda_{E x c}: 420-480 \mathrm{~nm}, \lambda_{E m}$ : $>515 \mathrm{~nm}$ ).

\section{Zusammenfassung}

Im Rahmen dieser Arbeit wurden sowohl pHsensitive, fluoreszente Nanopartikel, als auch $\mathrm{pH}$-insensitive Referenzpartikel synthetisiert. Diese Partikel wurden in eine Polyhydroxyethylmethacrylatmatrix integriert und in Kanäle eines mikrofluidischen Chips eingebracht sowie an eine im Mikrochip integrierte nanoporöse Glasmatrix gebunden. Die pH-Aufnahme erfolgte über Lumineszenzlebenszeitmessungen in der Frequenzdomäne. Es konnte gezeigt werden, dass mit diesen Sensormatrizen eine online Überwachung des lokalen pH-Wertes in mikrofluidischen Kanälen möglich ist.

\section{Ausblick}

Im weiteren Verlauf dieser Arbeit soll ein enzymatischer Mikrobiosensor entwickelt werden, welcher sich zur schnellen und mobilen Überwachung von Pestiziden eignet. Die Detektion von Pestizidrückständen soll dabei über die Aktivität des Enzyms AChE durch die lokale pH-Änderung erfolgen. Dafür soll das Enzym mit Hilfe eines Linkers kovalent an eine nanoporöse Glasmembran gebunden werden und die so modifizierte Membran in einen mikrofluidischen Chip integriert werden (Abb. 7).

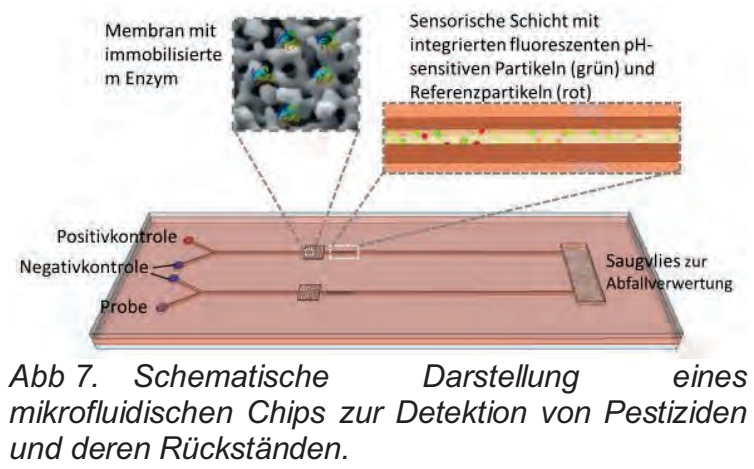

Die Echtzeitanalytik vieler Substanzen mithilfe solcher enzymatischer Biosensoren ist unter anderem für die flexible Umwelt- und Nahrungsmittelüberwachung von großem Interesse.

\section{Referenzen}

[1] C. Huber, I. Klimant, C. Krause, O. S. Wolfbeis, Dual Lifetime Referencing as Applied to a Chloride Optical Sensor, Analytical Chemistry 9, 2097-2103 (2001) ; doi: 10.1021/ac9914364.

[2] R. I. Nooney, E. McCormack, C. McDonagh, Optimization of size, morphology and colloidal stability of fluorescein dye-doped silica NPs for application in immunoassays, Analytical and Bioanalytical Chemistry 10, 2807-2818 (2012); doi: 10.1007/s00216-012-6224-z.

[3] J. M. Kürner, I. Klimant, C. Krause, H. Preu, W. Kunz, O. S. Wolfbeis, Inert Phosphorescent Nanospheres as Markers for Optical Assays, Bioconjugate Chem 6, 883-889 (2001); doi: 10.1021/bc000130x. 